\title{
Cancer Is Treatable via the Alternative Cellular Energy (ACE) Pathway
}

\author{
W. John Martin \\ Institute of Progressive Medicine, South Pasadena, CA, USA \\ Email: wjohnmartin@ccid.org
}

How to cite this paper: Martin, W.J. (2017) Cancer Is Treatable via the Alternative Cellular Energy (ACE) Pathway. Journal of Cancer Therapy, 8, 1279-1290. https://doi.org/10.4236/jct.2017.813106

Received: December 7, 2017

Accepted: December 25, 2017

Published: December 28, 2017

Copyright $\odot 2017$ by author and Scientific Research Publishing Inc. This work is licensed under the Creative Commons Attribution-NonCommercial International License (CC BY-NC 4.0). http://creativecommons.org/licenses/by-nc/4.0/ (c) (i) \&) Open Access

\begin{abstract}
Energy is required for cell replication, differentiation and specialized cellular processes, including cell death from apoptosis. The alternative cellular energy (ACE) pathway is an additional source of cellular energy beyond that obtained from the metabolism of food. The added energy is presumptively derived from an environment force defined as KELEA (kinetic energy limiting electrostatic attraction). The ACE pathway is expressed as a dynamic (kinetic) activity of the body's fluids that can seemingly facilitate biochemical reactions. Cancer is viewed as a metabolic derangement in genetically altered cells, in which the tumor cells have sufficient energy to replicate but insufficient cellular energy (ICE) to undergo preemptive apoptosis. Empirical observations are consistent with the premise that supplying additional cellular energy via the ACE pathway can enable tumor cells to undergo more timely apoptosis. The body's ACE pathway can be enhanced by administering either KELEA activated water or KELEA attracting compounds, referred to as enerceuticals ${ }^{\text {Th }}$. Increased levels of KELEA can also be created using various devices, which attract and subsequently transfer KELEA to the body or to water for subsequent administration. Several KELEA attracting devices operate by repetitive on-off electrical switching, while others involve the convergence of intermittent light paths. The fluctuating electrical activity of the brain is proposed to function as a variable antenna for bringing KELEA into the body. Preliminary evidence suggests that ACE pathway based interventional therapies may improve this presumed KELEA antenna function of the brain, leading to a sustainable improvement in the patient's ACE pathway. It is proposed that cancers are treatable and probably also preventable using methods to enhance the ACE pathway. Such methods may include the routine drinking of KELEA activated water.
\end{abstract}

\section{Keywords}

Cancer, ACE Pathway, KELEA, Enerceuticals, Stealth Adapted Viruses, 
Apoptosis, Homeopathy, Orgone, HANSI, Enercel, Dipolar, Herpes, Autism, Phototherapy

\section{Introduction}

Conventional therapies of non-surgically removable cancers include chemotherapy, radiation therapy, and immunotherapy. With few exceptions, these therapies lead to coincidental damage of normal cells, commonly causing considerable accompanying toxicity. Spontaneous regressions of cancers occasionally occur [1]. Such spontaneous regressions are generally attributed to the immune system [2]. This explanation assumes that tumor cells express cell surface antigens that can be recognized by antigen-specific cytotoxic T lymphocytes (CTL) [3]. Partial validation for this assumption is being provided by advanced molecular studies on cell surface proteins of several types of tumors [4]. The potential effectiveness of cancer immunotherapy is also consistent with promising clinical results using methods designed to overcome an apparent tumor-mediated "checkpoint" suppression of CTL [5].

A competing concept for tumor regression is the delayed triggering of an intrinsic process of cell death termed apoptosis [6]. According to this hypothesis, cells that acquire certain genetic changes, including those which lead to excessive growth and/or delayed maturation, will normally self-destruct via apoptosis. It is reasoned, therefore, that tumor growth may fundamentally reflect impaired apoptosis [7] [8] [9]. This hypothesis is supported by evidence that certain oncogenic viruses code for proteins that are inhibitory to apoptosis [10]. The failure of apoptosis by tumor cells is not likely to be absolute, but apoptosis may be sufficiently impaired to allow for the net overall growth of the tumor. Cellular energy is required for the biochemical processes involved in apoptosis. The provision of added cellular energy to cancer cells may overcome the existing metabolic barriers to apoptosis and, thereby, lead to tumor cell death.

Stealth adapted viruses evade effective immune recognition by the deletion or mutation of genes coding for the relatively few components normally targeted by the cellular immune system [11] [12] [13]. Studies on stealth adapted viruses led to the identification of particulate cellular materials, which can largely reverse the virus-induced cytopathic (cell damaging) effects (CPE) [14]. Furthermore, cells containing these materials remained viable despite the marked disruption of their mitochondria [15], the main supplier of cellular energy from the metabolism of food. It was proposed that the particulate materials were providing an alternative (non-mitochondria) source of cellular energy. Since the materials were usually pigmented, they were termed alternative cellular energy (ACE) pigments [14]. The reversal of stealth adapted virus-induced CPE was also achieved by adding a homeopathic (activated water) product to the re-feeding culture medium. These and other studies led to the realization that ACE pigments primarily 
function by altering the physical and chemical qualities of water. As described below, similar physiochemical changes can be induced in water treated with many compounds, including several products with reported benefits to human health, including improving the survival of cancer patients.

Extensive studies on the ACE pathway over the last several years have provided strong support for the proposal that a fundamental force exists, which prevents the fusion of electrostatically attracted opposing electrical charges [13] [16]-[21]. The force is termed KELEA (kinetic energy limiting electrostatic attraction). KELEA is seemingly attracted to separated electrical charges, including the opposing electrical charges on dipolar molecules. When present in water, KELEA apparently leads to a loosening of intermolecular hydrogen bonding between water molecules [16]. KELEA induced water activation was initially characterized as reduced surface tension, increased volatility and striking linear dissolving patterns of neutral red dye particles sprinkled onto the water. Additional defining properties of KELEA activated water will be detailed in forthcoming publications. KELEA can similarly affect other fluids, including ethanol and gasoline [16] [22] [23]. Moreover, KELEA leads to a higher energy yield from the combustion of gasoline. This is consistent with KELEA being a primary source of chemical energy [24].

While the ACE pathway overlaps with food metabolism in promoting various cellular activities, it can support cellular functions beyond those provided by food [25]. Specifically, the ACE pathway provides an effective non-immunological defense against viral and bacterial infections [26]-[31]. It can dramatically reduce scarring from acute trauma, including burns [32] and improves the healing of chronic wounds [32]. Clinical observations also suggest that enhancing the ACE pathway can lead to sustainable improvements in certain brain functions. Included in the improved neurological activities may be the capacity of the brain to function as an antenna for KELEA and, thereby, to act as a natural driver of the body's ACE pathway [33] [34] [35]. The focus of this paper is the potential role of the ACE pathway as a source of cellular energy to promote the regression of tumors, presumptively via apoptosis.

As discussed in the next section, there is an overlap between the methods, which apparently can transfer KELEA into water and currently and/or historically promoted remedies and energy-based devices used for treating cancer patients. The overlap is consistent with the previously offered proposal that the clinical effectiveness of many Complementary and Alternative Medicine (CAM) modalities is mediated via strengthening of the ACE pathway [36] [37] [38] [39].

\section{Water Activating Methods}

Water can be activated with KELEA by either adding various compounds to the water or by exposing the water to KELEA-attracting energy fields. Once the water is sufficiently activated, the activating compounds can be removed from the water. This can be done by decanting the water from insoluble compounds, or 
for soluble compounds by either repeated dilutions of the water, as in homeopathy, or by zero-residue filtration. Similarly, water remains activated after being removed from water-activating energy fields.

One grouping of substances with postulated benefits to cancer patients is mineral containing compounds. These includes humic/fulvic acids, zeolites, bentonite, kaolin, mica, magnesium oxide, a range of other metallic oxides, germanium, tourmaline, shungite (a carbon-based product from Russia), volcanic rock materials, etc., [36] [37] [38] [39] These products are usually marketed as sources of essential minerals, which are arguably deficient in some cancer patients. Other natural compounds being marketed to cancer patients include vitamins, tinctures, essential oils and herbal products. These compounds are variously promoted for their anti-oxidant, metal-chelating, anti-inflammatory and/or presumed immune-enhancing activities. Many of the above-mentioned compounds have now been tested for the ability to activate water, as assessed by increasing the water volatility. Although the findings are preliminary and the levels of activation are variable and dependent upon concentrations and durations of exposure, it is concluded that a very wide range of compounds can lead to some degree of water activation. The more effective compounds are being referred to as enerceuticals ${ }^{\mathrm{Tm}}$.

A consistent feature of water activating compounds is the spatial separation of positive and negative electrically charged regions, such that the compounds are functionally dipolar. Ionized atoms comprising cations and anions are also intrinsically dipolar because they differ in the relative numbers of protons and electrons. Cations have one to four more protons than electrons, while anions have more electrons than protons. The excess charges can be offset by ionic bonding between cations and anions, especially if not highly diluted in the water. The lanthanide series of rare earth minerals are notable in that their cations can have up to four fewer electrons in the $4 \mathrm{f}$ orbital, while retaining electrons in the outer $5^{\text {th }}$ orbital. The outer electrons tend to shield some of the positive charge from anion neutralization [40]. Known water activating rare earth minerals include neodymium and cerium. Electrolysis, including the use of electromagnetic energy-generated electrical plasma, can also be used to separate positive from negative ions. Electrolysis can also generate non-thermally vaporized, activated water molecules, which are present along with oxygen and hydrogen gases in material referred to as Brown's gas [41].

Water activating energy fields can be created with various devices related to those previously proposed as being useful in cancer therapy. Several of the devices utilize rapid on-off electrical switching. Prominent early pioneers in the development of these types of devices include Nikola Tesla (radiant energy), Georges Lakhovsky (multiwave oscillator), Royal Raymond Rife (beam ray) and Panos Pappas (papimi machine) [36] [37] [38] [39]. Igor Smirnov currently employs a fluctuating light as a means of oscillating magnetically aligned molecules in producing MRET (Molecular Resonance Effect Technology) water [42] [43]. 
His method is similar in principle to that of using radio waves in nuclear magnetic resonance. Another currently used technique developed by Robert Religa and Sandra Michael, involves using sets of facing computers with matching displays of moving lines of colored images. The light projected from the computer screens converges onto common midpoints within the room. Some other earlier pioneers pursued seemingly more stable sources of energy, such as cobalt blue glass (Augustus Pleasonton), static electricity (d'Arsonval's galvanic cage) and "orgone" chambers (Wilhelm Reich) [44]. The use of cobalt oxide in glass has been extended to currently include additional mineral oxides, e.g. in Miron glass bottles (miron-glas.com). Wilhelm Reich identified orgone as a universal force with biological and biophysical properties. Elevated levels of orgone develop in chambers with walls composed of alternating layers of electrical conducting and insulating materials. Cancer patients would be treated by sitting within such chambers. Subsequent followers of Reich's work argue that they can attract and transmit orgone using pyramids of polyether resins, which are embedded with dispersed mixed minerals, metal coils and specifically oriented quartz and other crystals. These pyramids are able to somewhat activate water. So too can certain crystals directly lead to water becoming activated [45]. Resins and other containers are being used to embed various water-activating ceramics to avoid any leaching of the chemicals into the water. Direct contact of the water with the activating compounds is, therefore, not a necessary requirement for activating water.

Another indication of distant activation of water is provided by the Aquapol device developed by Wilhelm Mohorn. This device reverses the upward seepage of water into the foundations of buildings [46]. It consists of a coil with attached antennas and an energy gathering metal plate. It is positioned onto the ceiling of a room, yet affects the groundwater moving up the foundations of the building. Negative ions are said to be increased around the device (personal communication).

Johann Grander also realized that the activated water, which he obtained from deep within an old copper mine in Austria, could be used to indirectly activate other water. His commercial water activating device comprises an inner compartment into which he placed some of the active water from the copper mine. Regular water would flow into the device without directly entering the inner compartment [47]. The water emerging from the device acquires substantial beneficial qualities in various biological and industrial applications. This and related observations carry the important inference that consuming or even being exposed to activated water can potentially induce biophysical effects throughout the body, ostensibly via the secondary activation of the body's ACE pathway.

Direct evidence for the above-stated inference was obtained in studies employing neutral red dye dissolved in activated fluid. Activated water with dissolved neutral red dye will show a striking fluorescence when illuminated with ultraviolet (UV) light. This is not seen with regular water at normal $\mathrm{pH}$. Enhanced UV fluorescence with added dye is also seen upon activation of some 
other fluids. A protocol was developed comprising the placing a solution of neutral red dye in an activated fluid over skin lesions caused by either herpes simplex virus (HSV) or herpes zoster virus (HZV). Illuminating the solution with UV light results in the underlying lesion becoming fluorescent when directly viewed with UV light. This transfer of energy is presumably to the ACE pigments within the herpes skin lesion. The energy transfer occurs even with intervening light impermeable material between the illuminated solution and the underlying herpes skin lesion [26] [27]. A similar protocol was tested in children with autism. Rather than targeting specific skin lesions, the UV illuminated solution that is applied to paper towels or contained in a sealed Ziploc pouch, was placed over areas of the child's back or onto the soles of the child's feet. Again, this procedure induced direct UV fluorescence in the underlying skin of the child, as well as direct UV fluorescence in other regions of the child's skin and within the oral cavity [48]. Again, the induction of direct skin fluorescence occurred even with an intervening layer of light impermeable black plastic. The inducible skin fluorescence would slowly fade over several hours. Parents noted definite improvements in their autistic children using this protocol.

\section{Homeopathy Is KELEA Activated Water}

The practice of homeopathy [49] is based on a false premise of specificity, as implied in the Law of Similars [50]. This Law asserts that each specific symptom in a patient can be alleviated by administering a highly dilute solution of a compound that induces the exact same symptom, when administered in high concentrations to normal, healthy individuals. Homeopathy also assumes that the compound can be so diluted in water as to be no longer physically present, but to have left a "memory" in the water that is unique for the compound. No crossover study has been published that convincingly proves that there is specificity of symptom relief using different formulations under controlled "double-blind" conditions. Indeed, clinical experience confirms that certain homeopathic formulations are clinically effective in multiple clinical conditions (discussed below).

Homeopathic remedies are typically produced by using alcoholic extracts (tinctures) of materials from one or more plants, with or without added minerals. Each formulation is diluted into water or into water with a low content of ethanol. Additional 10 to 100 -fold dilutions are subsequently made, with the solution being jolted (succussed) between dilutions and before administration. Mainly for proprietary reasons, the actual ingredients in many homeopathic formulations are rather vaguely described and not always honestly listed. Different homeopathic remedies are intended to be administered by injections, inhalation, orally or topically.

The removal of specificity as an activity parameter of homeopathic remedies, leads to the conclusion that the products simply comprise water, which has acquired biological activity during the repetitive dilutions of various tinctures. This approach deemphasizes the chemical formulation of the activating tinctures. A well standardized, homeopathic product is called Enercel. It is related to a product 
developed in Argentina called HANSI (homeopathic activator of the natural system immune). Both HANSI and Enercel have been used to treat cancer patients over several years. The reported benefits of HANSI in pancreatic cancer patients [51] and of Enercel in breast, lung and renal cancer patients [29] are impressive. The major modes of administration of HANSI and Enercel have been by intravenous and intramuscular injections. Consideration has also been given to injections of Enercel into acupuncture points [52] and directly into tumors. Of note, is the absence of pain during Enercel-induced tumor regression. This finding is consistent with apoptosis being the primary mechanism of tumor cell death. Enercel has also been shown to be effective in treating other illnesses, including tropical diarrhea [30], HIV [28], tuberculosis [28] and amyotrophic lateral sclerosis [52]. Enercel was also effective in achieving scar-free healing of a skin burn, which would otherwise have required skin grafting [32].

\section{Future Studies}

The major goal of future studies is to devise a method for accurately assessing the ACE pathway in cancer patients. The method can help optimize efforts to maximally enhance the ACE pathway in these patients. Moreover, a better quantitation of in vivo efficacy of various therapeutic approaches to enhancing the ACE pathway will help shed light on the nature of KELEA and the proposed capacity of the brain to act as a natural antenna for KELEA. Another important goal is to confirm the ability of KELEA activated fluids and/or KELEA enhanced environments to induce apoptosis in tumor and not in normal cells. These studies can be initially performed on cultured tumor and non-tumor cell lines. Cell growth can be monitored using metabolic markers, such as MTT (3-(4,5-dimethylthiazol-2-yl)-2,5-diphenyl tetrazolium bromide) [53]. Molecular markers of apoptosis include terminal deoxynucleotidyl transferase dUTP nick end labeling (TUNEL) [54].

These assays will help in the selection and further optimization of KELEA activated fluids. Enercel is currently undergoing clinical trials in cancer patients in Ukraine. In general, patients are being offered daily intravenous injections of 50 $\mathrm{ml}$ Enercel over the period of a month. In addition to evidence of tumor regressions, a consistent finding is the self-reported improvements in emotional wellness, which are persisting beyond the period of Enercel administration. Tentatively, Enercel administration appears to be augmenting the brain's proposed role as a natural antenna for KELEA. If so, this benefit can potentially lead to continuing, sustainable support for the ACE pathway.

To date, most cancer patients receiving non-conventional therapies have been at later stages of their illness, having had one or more prior courses of chemotherapy. It would seem preferable to begin ACE-pathway based protocols immediately after the initial cancer diagnosis. Detailed molecular analyses on biopsied tumor tissue can take up to several weeks. This interval provides a window of opportunity to assess any possible reduction in tumor size achievable using ACE 
pathway based anti-tumor therapies. Positive findings could reasonably then lead to an extension of the delay in instigating chemotherapy. Suitable patients for investigative, first-line ACE pathway based therapy, can also include patients who are motivated by the adverse outcomes of conventionally treated relatives. Success in ACE pathway-based cancer therapy will quickly lead to consideration of the role of the ACE pathway in cancer prevention and, indeed, to the many other benefits of routinely consuming KELEA activated fluids.

\section{Conclusions}

The continuing growth of tumors is attributed to the tumor cells having insufficient cellular energy (ICE) to undergo death by apoptosis. It is reasoned that if additional cellular energy was available, the tumor cells will overcome the barrier(s) to apoptosis and self-destruct. KELEA (kinetic energy limiting electrostatic attraction) is perceived as a fundamental force that prevents the fusion of electrostatically attracted opposing electrical charges. KELEA can add to the kinetic and chemical energy of water molecules and other substances. KELEA activation of intercellular and extracellular water within living organisms provides a source of cellular energy beyond that obtained from the metabolism of food. The additional energy provides an alternative cellular energy (ACE) pathway for a range of cellular activities, some of which are not supported by increased food consumption. A likely distinguishing function of the ACE pathway is facilitating tumor cell apoptosis. Another distinguishing characteristic may be ACE pathway mediated improvements in aspects of brain functions, including the brain's capacity to function as an antenna to attract KELEA into the body. The ACE pathway can also provide a non-immunological defense mechanism against various pathogens, potentially including oncogenic viruses.

The administration of KELEA activated water is discussed as a practical method for enhancing the ACE pathway. Studies are underway comparing intravenous administration with oral consumption, inhalation and widespread, direct skin exposure. KELEA activated water can be produced using two basic methods. One method involves the addition of selected dipolar compounds to water. These compounds can be subsequently removed from the activated water. The second approach is to place the water in an environment with increased levels of KELEA. These environments can be created using various devices, some of which use repeated on-off electrical switching. Using these methods, large volumes of activated water can be inexpensively produced.

Clinical observations on the use of Enercel as an injectable solution support the concept of induced tumor regression with minimal inflammation, consistent with ACE pathway induced apoptosis. Other modes of Complementary and Alternative Medicine (CAM) modalities are similarly consistent with activating the body's ACE pathway as cancer therapy. The effects of KELEA activated and control tissue culture media on the growth and survival of cultured normal and tumor cells can provide additional insights into the ACE pathway. Clinical testing 
of KELEA activated water should be provided as first-line therapy in cancer patients.

\section{Acknowledgements}

The Institute of Progressive Medicine is a component of MI Hope Inc., a nonprofit public charity. Dr. David Christner of World Health Advanced Technologies, Ltd., kindly provided clinical information relating to the use of Enercel in cancer patients. Clinicians interested in testing KELEA activated water and KELEA generating medical devices as possible cancer therapies can directly contact the author. So too may basic researchers willing to assist in studies on KELEA.

\section{Conflict of Interest}

None.

\section{References}

[1] Challis, G.B. and Stam, H.J. (1990) The Spontaneous Regression of Cancer. A Review of Cases from 1900 to 1987. Acta Oncologica, 29, 545-550. https://doi.org/10.3109/02841869009090048

[2] Cole, W.H. (1981) Efforts to Explain Spontaneous Regression of Cancer. Journal of Surgical Oncology, 17, 201-209. https://doi.org/10.1002/jso.2930170302

[3] Vigneron, N. (2015) Human Tumor Antigens and Cancer Immunotherapy. BioMed Research International, 2015, Article ID: 948501. https://doi.org/10.1155/2015/948501

[4] Tran, E., Robbins, P.F. and Rosenberg, S.A. (2017) "Final Common Pathway" of Human Cancer Immunotherapy: Targeting Random Somatic Mutations. Nature Immunology, 18, 255-262. https://doi.org/10.1038/ni.3682

[5] Chowdhury, P.S., Chamoto, K. and Honjo, T. (2017) Combination Therapy Strategies for Improving PD-1 Blockade Efficacy: A New Era in Cancer Immunotherapy. Journal of Internal Medicine, E-pub ahead of print. https://doi.org/10.1111/joim.12708

[6] Elmore, S. (2007) Apoptosis: A Review of Programmed Cell Death. Toxicologic Pathology, 35, 495-516. https://doi.org/10.1080/01926230701320337

[7] Fernald, K. and Kurokawa, M. (2013) Evading Apoptosis in Cancer. Trends in Cell Biology, 23, 620-633. https://doi.org/10.1016/j.tcb.2013.07.006

[8] Mohammad, R.M., Muqbil, I., Lowe, L., Yedjou, C., Hsu, H.-Y., Lin, L.-T., et al. (2015) Broad Targeting of Resistance to Apoptosis in Cancer. Seminars in Cancer Biology, 35, S78-103. https://doi.org/10.1016/j.semcancer.2015.03.001

[9] Shimizu, S. (2015) Development of Anti-Cancer Drugs Mediated by Apoptosis and Autophagy. Nihon Rinsho, 73, 1302-1307.

[10] Fuentes-Gonzalez, A.M., Contreras-Paredes, A., Manzo-Merino, J. and Lizano, M. (2013) The Modulation of Apoptosis by Oncogenic Viruses. Virology Journal, 10, 182. https://doi.org/10.1186/1743-422X-10-182

[11] Martin, W.J. (1994) Stealth Viruses as Neuropathogens. CAP Today, 8, 67-70.

[12] Martin, W.J. (2014) Stealth Adaptation of Viruses: Review and Updated Molecular Analysis on a Stealth Adapted African Green Monkey Simian Cytomegalovirus 
(SCMV). Journal of Human Virology \& Retrovirology, 1, 00020. https://doi.org/10.15406/jhvrv.2014.01.00020

[13] Martin, W.J. (2014) Stealth Adapted Viruses; Alternative Cellular Energy (ACE) \& KELEA Activated Water. Author House, Bloomingdale, IN, p. 321.

[14] Martin, W.J. (2003) Stealth Virus Culture Pigments: A Potential Source of Cellular Energy. Experimental and Molecular Pathology, 74, 210-223. https://doi.org/10.1016/S0014-4800(03)00037-6

[15] Martin, W.J. (2003) Complex Intracellular Inclusions in the Brain of a Child with a Stealth Virus Encephalopathy. Experimental and Molecular Pathology, 74, 179-209.

[16] Martin, W.J. (2015) KELEA: A Natural Energy that Seemingly Reduces Intermolecular Hydrogen Bonding in Water and Other Liquids. Open Journal of Biophysics, 5, 69-79. https://doi.org/10.4236/ojbiphy.2015.53006

[17] Martin, W.J. (2015) Improved Efficiency of Heat Exchange Using KELEA Activated Water. Open Journal of Energy Efficiency, 4, 36-43. https://doi.org/10.4236/ojee.2015.42004

[18] Martin, W.J. (2016) KELEA (Kinetic Energy Limiting Electrostatic Attraction) May Add to the Measured Weight of an Object. Journal of Modern Physics, 7, 461-472. https://doi.org/10.4236/jmp.2016.76048

[19] Martin, W.J. (2016) KELEA (Kinetic Energy Limiting Electrostatic Attraction) Offers an Alternative Explanation to Existing Concepts Regarding Wave-Particle Duality, Cold Fusion and Superconductivity. Journal of Modern Physics, 7, 1995-2007. https://doi.org/10.4236/jmp.2016.715176

[20] Martin, W.J. (2015) Interacting Light Paths Attract KELEA (Kinetic Energy Limiting Electrostatic Attraction) and Can Lead to the Activation of Water. Open Journal of Biophysics, 5, 115-121.

[21] Martin, W.J. (2015) Interactive Electric Fields Can Attract KELEA (Kinetic Energy Limiting Electrostatic Attraction) and Can Lead to the Activation of Water. International Journal Complementary \& Alternative Medicine, 1, Article ID: 00034. https://doi.org/10.15406/ijcam.2015.01.00034

[22] Martin, W.J. (2016) KELEA (Kinetic Energy Limiting Electrostatic Attraction) Can Markedly Improve the Performance of Gasoline and Diesel Fuels in Power Generation and in Transportation. Journal of Transportation Technologies, 6, 148-154. https://doi.org/10.4236/jtts.2016.63014

[23] Martin, W.J. (2017) Using KELEA (Kinetic Energy Limiting Electrostatic Attraction) to Improve the Efficiency of Fuel Combustion. Open Journal of Air Pollution, 6, 103-116. https://doi.org/10.4236/ojap.2017.63009

[24] Martin, W.J. (2017) Is KELEA (Kinetic Energy Limiting Electrostatic Attraction) A Source of Chemical Energy? MOJ BiorgInorgChem, 1, Article ID: 00011. https://doi.org/10.15406/mojboc.2017.01.00011

[25] Martin, W.J. (2017) The Many Biological Functions of the Alternative Cellular Energy (ACE) Pathway. International Journal of Complementary \& Alternative Medicine, 7, Article ID: 00237. https://doi.org/10.15406/ijcam.2017.07.00237

[26] Martin, W.J. and Stoneburner, J. (2005) Symptomatic Relief of Herpetic Skin Lesions Utilizing an Energy-Based Approach to Healing. Experimental and Molecular Pathology, 78, 131-134. https://doi.org/10.1016/j.yexmp.2004.10.007

[27] Martin, W.J. and Stoneburner, J. (2014) Alternative Cellular Energy (ACE) Pathway Activation as the Mode of Action of Neutral Red Dye Phototherapy of Human Viruses. Journal of Human Virology \& Retrovirology, 1, Article ID: 00019.

[28] Dubrov, V., Dubrova, T., Christner, D., Laurent, D. and Martin, W.J. (2015) Alter- 
native Cellular Energy Based Therapy using Enercel in Advanced AIDS Patients Co-Infected with Tuberculosis and Treated in Chernigov, Ukraine. Journal of Human Virology \& Retrovirology, 2, Article ID: 00061.

[29] Martin, W.J. and Laurent, D. (2015) Homeopathy as a Misnomer for Activation of the Alternative Cellular Energy Pathway: Evidence for the Therapeutic Benefits of Enercel in a Diverse Range of Clinical Illnesses. International Journal of Complementary \& Alternative Medicine, 2, Article ID: 00045. https://doi.org/10.15406/ijcam.2015.02.00045

[30] Izaguire, R.R., Guzman, M.R., Fuentes, R.C., Mena, C.E., Penate, E., et al. (2014) Alternative Cellular Energy Based Therapy of Childhood Diarrhea. In: Stealth Adapted Viruses, Alternative Cellular Energy (ACE) and KELEA Activated Water, Author House, Bloomingdale, IN, 103-114.

[31] Martin, W.J. (2016) The ACE Pathway in Comparison to the Immune System in the Defense against Infectious Diseases. Journal of Human Virology \& Retrovirology, 4, Article ID: 00124.

[32] Martin, W.J. (2017) Tissue Regeneration without Scarring Achieved by Enhancing the Alternative Cellular Energy (ACE) Pathway. Journal of Cosmetics, Dermatological Sciences and Applications, 7, 82-98. https://doi.org/10.4236/jcdsa.2017.71009

[33] Martin, W.J. (2017) Insufficiency of Cellular Energy (ICE) from the Alternative Cellular Energy (ACE) Pathway Limiting the Specialized Functions of Neuronal Cells. International Journal of Complementary \& Alternative Medicine, 4, Article ID: 00112.

[34] Martin, W.J. (2017) Insufficiency of Cellular Energy (ICE) in Neurons: From Electrical Hyperactivity to Quiescence. International Journal of Complementary \& Alternative Medicine, 4, Article ID: 00118.

[35] Martin, W.J. (2017) Hyper-Excitability Followed by Functional Quiescence in Neuronal Cells Caused by Insufficient Cellular Energy (ICE): Treatable by Enhancing the Alternative Cellular Energy (ACE) Pathway. World Journal of Neuroscience, 7, 257-266. https://doi.org/10.4236/wjns.2017.73021

[36] Martin, W.J. (2016) Cancer as an Insufficiency of Cellular Energy (ICE): Therapeutic Approaches Based on Enhancing the Alternative Cellular Energy (ACE) Pathway. International Journal Complementary Alternative Medicine, 3, Article ID: 00074. https://doi.org/10.15406/ijcam.2016.03.00074

[37] Martin, W.J. (2015) KELEA Activation of Water and Other Fluids for Health, Agriculture and Industry. Journal of Water Resource and Protection, 7, 1331-1344. https://doi.org/10.4236/jwarp.2015.716108

[38] Martin, W.J. (2015) Alternative Cellular Energy Pathway Therapy using KELEA Activated Water. International Journal of Complementary \& Alternative Medicine 2, Article ID: 00051. https://doi.org/10.15406/ijcam.2015.02.00051

[39] Martin, W.J. (2015) Alternative Cellular Energy. A Unifying Concept in Complementary Alternative Medicine. International Journal of Complementary \& Alternative Medicine, 1, Article ID: 00022.

[40] Azimi, G., Dhiman, R., Kwon, H.-M., Paxson, A.T. and Varanasi, K.K. (2013) Hydrophobicity of Rare-Earth Oxide Ceramics. Nature Materials, 12, 315-320. https://doi.org/10.1038/nmat3545

[41] Hurtak, J.J. and Hurtak, D. (2014) The History and Future of Brown's Gas. Nexus Magazine, 21, 45-54.

[42] Smirnov, I.V. (2007) MRET Activated Water and Its Successful Application for Preventive Treatment and Enhanced Tumor Resistance in Oncology. European Journal for Scientific Research, 16, 575-583. 
[43] Vysotskii, V.I., Smirnov, I.V. and Kornilova, A.A. (2005) Introduction to the Biophysics of Activated Water. Universal Publishers, Boca Raton, 133.

[44] Reich, W. (1948) The Cancer Biopathy. In: The Discovery of the Orgone, Translated by White A, Higgins M and Raphael CM (1973), Vol. 2, Farrar, Straus and Giroux, New York, 433.

[45] Gienger, M. and Goebel, J. (2007) Gem Water. Earthdancer GmbH, 94.

[46] Aquapol Hellas (2012). https://issuu.com/aquapolhellas/docs/aquapol

[47] http://www.grandertechnology.com

[48] Martin, W.J. (2014) Alternative Cellular Energy (ACE) Pathway Activation as Natural Therapy for Autism. In: Stealth Adapted Viruses, Alternative Cellular Energy (ACE) and KELEA Activated Water, Author House, Bloomingdale, IN, 87-102.

[49] Marcy, E.E. and Hunt, F.W. (1868) The Homeopathic Theory and Practice of Medicine. William Radde, New York, 942.

[50] Gutman, W. (1961) The Basis of the Law of Similars. British Homoeopathic Journal, 50, 26-29. https://doi.org/10.1016/S0007-0785(61)80078-1

[51] Bertacchini, C. (1992) The Effects of Homeopathic Formulas Treatment on Aspects of the Quality of Life in Patients with Advanced Pancreatic Cancer. http://www.bryomixol.com/estudios/THE_FFECTS.pdf

[52] Liang, S., Christner, D., Du Laux, S. and Laurent, D. (2011) Significant Neurological Improvement in Two Patients with Amyotrophic Lateral Sclerosis after 4 Weeks of Treatment with Acupuncture Injection Point Therapy using Enercel. Journal of Acupuncture \& Meridian Studies, 4, 257-261.

https://doi.org/10.1016/j.jams.2011.09.017

[53] Hayon, T., Dvilansky, A., Shpilberg, O. and Nathan, I. (2003) Appraisal of the MTT-Based Assay as a Useful Tool for Predicting Drug Chemosensitivity in Leukemia. Leuk Lymphoma, 44, 1957-1962. https://doi.org/10.1080/1042819031000116607

[54] Loo, D.T. (2011) In Situ Detection of Apoptosis by the TUNEL Assay: An Overview of Techniques. Methods in Molecular Biology, 682, 3-13. https://doi.org/10.1007/978-1-60327-409-8_1

\section{Abbreviations}

ACE: Alternative Cellular Energy

CPE: Cytopathic Effect

CTL: Cytotoxic T Lymphocytes

CAM: Complementary and Alternative Medicine

ICE: Insufficient Cellular Energy

KELEA: Kinetic Energy Limiting Electrostatic Attraction

UV: Ultraviolet

HSV: Herpes Simplex Virus

HZV: Herpes Zoster Virus 